\title{
Alkylative Ring-Opening of Bicyclic Aziridinium Ion and Its Application for Alkaloid Synthesis
}

\author{
Nagendra Nath Yadav ${ }^{1}$, Young-Gun Lee ${ }^{2}$, Nikhil Srivastava ${ }^{2}$ and Hyun-Joon $\mathrm{Ha}^{2 *}$ \\ ${ }^{1}$ Department of Chemistry, North Eastern Regional Institute of Science and Technology, Nirjuli, India, ${ }^{2}$ Department of \\ Chemistry, Hankuk University of Foreign Studies, Yongin, South Korea
}

Alkylative ring-opening of bicyclic aziridinium ion generated from 4-hydroxybutylaziridine with organocopper reagent was achieved successfully to afford 2-alkylsubstituted piperidine in high or moderate yield. This method allowed carbon-carbon bond formation of "non-activated" aziridine via aziridinium ion ring-opening in regio- and stereo-selective manner for the first time. This newly developed reaction was applied for an efficient synthesis of alkaloid with the representative example of conine and epiquinamide.

Keywords: aziridine, bicyclic aziridinium ions, alkylation, ring-expansion, alkaloid synthesis

\section{INTRODUCTION}

OPEN ACCESS

Edited by:

Andrea Basso,

University of Genoa, Italy

Reviewed by:

Won Koo Lee,

Sogang University, South Korea Andrea Trabocchi,

University of Florence, Italy

*Correspondence:

Hyun-Joon $\mathrm{Ha}$

hiha@hufs.ac.kr

Specialty section: This article was submitted to

Organic Chemistry, a section of the journal

Frontiers in Chemistry

Received: 03 May 2019

Accepted: 11 June 2019

Published: 27 June 2019

Citation:

Yadav NN, Lee Y-G, Srivastava N and Ha H-J (2019) Alkylative Ring-Opening

of Bicyclic Aziridinium lon and lts

Application for Alkaloid Synthesis.

Front. Chem. 7:460.

do: 10.3389/fchem.2019.00460
Aziridine as a nitrogen-containing three membered ring has high ring-strain described as a "spring-loaded" compound by Yudin in his book (Yudin, 2006). This ring strain has two different aspects as unstability and high reactivity. Comparing to other popular three membered rings such as cyclopropane and oxirane (epoxide), aziridine has an extra-commander to control of two different aspects by the change of the characteristics of aziridine ring-nitrogen (Yudin, 2006). When the aziridine ring-nitrogen has electron-attracting substituent we call it "activated aziridine" with instability and high activity. Most of these aziridines are quite reactive toward the aziridinering opening with the most nucleophiles including carbon nucleophiles (Ghorai et al., 2011). However, so called "non-activated aziridine" (D’hooghe and Ha, 2016) with strong basic ring nitrogen having electron-donating substituents such as alkyl groups are not reactive toward either common alkyl nucleophiles or quite reactive heteroatom nucleophiles such as azide, acetate, alkoxide. For the successful reactions with these heteroatom nucleophiles, the aziridine ring should be activated as aziridinium ion in the presence of acids (Kim et al., 2006; Stanković et al., 2012). In past few years we succeeded in generation of alkyl aziridinium ions as their stable forms in inter- and intramolecular manner and their successful ring opening with various heteroatom nucleophiles to give multi-substituted nitrogen-containing valuables in high regio- and stereospecific manner (Kim et al., 2008; Dolfen et al., 2016; Boydas et al., 2018). However, limited number of alkylative aziridine-ring opening was succeed only after $\mathrm{N}$-methylation by highly reactive methyl triflate (Yoon et al., 2012).

In this communication we would like to report generation of 1-azoniabicyclo[4.1.0]heptanes tosylate as one of aziridinium ions and its regio- and stereo-specific ring opening for the synthesis of chain extended 2-alkylpiperidine. This chemistry has been used for efficient synthesis of alkaloids such as conine and epiquinamide as representative examples (Graphical Abstract). Efficient and stereoselective construction of aza-heterocycles have always been a challenge for synthetic organic chemists because they played a very important role in the field of pharmaceutical industry (Bailey et al., 1998; Husson and Royer, 1999; Passarella et al., 2002; Carry et al., 2013; Yadav et al., 2016). Among 


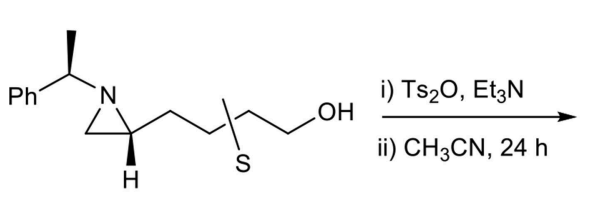

$S=$ Substituent

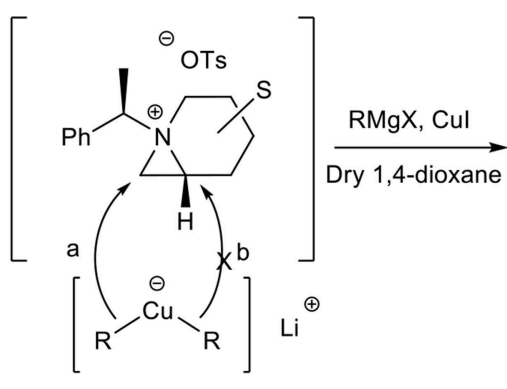

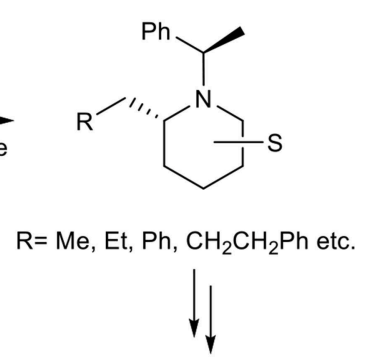

(+)-Conine and (+)-Epiquinamide

Graphical Abstract | Alkylative ring-opening of bicyclic aziridinium ion for alkaloid synthesis.

them piperidines as a six-membered aza-heterocycles are present in large number of biologically active natural products especially in the class of alkaloids. Many novel synthetic approaches have been developed for aza-heterocycles in an efficient manner to encounter regio-and stereoselective demand for the drug specification (Nicolaou et al., 1995; Masse et al., 2000; Kumar and Bodas, 2005; Trost et al., 2007; Srivastava and Panda, 2008; Chavan et al., 2015). However, no synthetic method is available to construct piperidine ring with concomitant introduction of the proper alkyl group at the alpha-position of the piperidine ring. Recently, we succeeded in regio- and stereoselective ring opening reaction of stable bicyclic aziridinium ion by diverse heteroatom nucleophiles to get various pipiridines and azepane in good to excellent yields (Ji et al., 2014; Eum et al., 2015; Dolfen et al., 2016; Choi et al., 2017; Yadav and Ha, 2018; Macha et al., 2019). In continuation of this chemistry, we herein report the alkylative, regio-, and stereo-selective ring-opening of 1azoniabicyclo[4.1.0] heptanes tosylate by various organocopper reagents to afford piperidines with carbon-chain extension at C2 position. This selective transformation allow us to access various biologically important natural products with representative examples including (+)-conine (Hattori and Yamamoto, 1993; Hirai and Nagatsu, 1994; Munchhof and Meyers, 1995; Reding and Buchwald, 1998), and (+)-epiquinamide (Suyama and Gerwick, 2006; Tong and Barker, 2006; Srivastava et al., 2009; Airiau et al., 2010) in highly efficient manner.

\section{RESULTS AND DISCUSSION}

The bioavailability and importance of 2-alkyl piperidines as key intermediate for synthesis of various bioactive compounds encourage us to develop an efficient method for these systems from chiral aziridines. Our recent report on formation of stable 1-azoniabicyclo[4.1.0] heptanes tosylate 2 and its subsequent ring opening by the heteroatom nucleophile to give heteroatomsubstituted piperidine and azepanes, promoted us to explore carbon-chain extension with concomitant highly strained aziridine-ring expansion with carbon nucleophile. Initially, we have treated freshly prepared bicyclic aziridinium ion 2 with $n$-PrMgBr in dry THF but the desired ring expanded product 3 was not formed. This may be due to hardness of the nucleophile generated from Grignard reagent (Kozlowski, 1991). Then we added CuI as reagent for in situ generation of organocopper reagents, which can changed the nature of nucleophile (Kozlowski, 1991) and we obtained the required product in 50\% yields (Scheme 1). Furthermore, the reaction is highly regioselective and ring-opening proceeded by nucleophilic attack at $\mathrm{C} 2$ of aziridinium ion $\mathbf{2}$ to give piperidine $\mathbf{3 a}$ along with formation of traces of azepane compound $\mathbf{4}$ by nucleophilic attack at $\mathrm{C} 3$.

Once we isolated and characterized compound $3 \mathbf{a}$, we tried to improve the regioselectivity and yields. We have screened several different $\mathrm{Cu}(\mathrm{I})$ salts and solvents to carry the reaction. We found that the reaction of bicyclic aziridinium tosylate 2 with $n-\mathrm{PrMgBr}$ in anhydrous 1,4-dioxane in the presence of $\mathrm{CuI}$ gave the desired product $\mathbf{3 a}$ in $70 \%$ yields (Table $\mathbf{1}$ ).

Once we have optimized reaction condition for alkylative ring opening of aziridinium ion by organocopper reagents in hand, we used various reagents generated in situ from Grignard reagents and $\mathrm{CuI}$ in 1,4-dioxane to get various piperidine $\mathbf{3} \mathbf{a}-\mathbf{l}$ as shown in Table 2. Reagents having non-hindered alkyl group such as $n$-propyl $\mathbf{3 a}$, methyl $\mathbf{3} \mathbf{f}$, ethyl $\mathbf{3} \mathbf{b}$, and allyl $\mathbf{3 e}$ resulted into the expected product in good yields $(>50 \%)$, while those having hindered and bulky alkyl groups such as benzyl 3c, n-nonyl 3d, phenyl 3g, iso-propyl 3i, $p$-chlorophenyl $3 \mathbf{k}$, and cyclopentyl $3 \mathbf{l}$ gave poor yields (25-38\%) due to possible hindrance for the alkyl nucleophiles to approach the aziridinium ions.

After successful development of a new method to prepare 2alkyl substituted piperidines from chiral aziridines, our attention was to apply this reaction for a short and efficient synthetic route for few biologically active molecules having piperidine skeleton such as $(+)$-conine 6 and $(+)$-epiquinamide 7 (Figure 1).

Optically active conine, the poisonous hemlock alkaloid is a very important among piperidines alkaloids (Hattori and Yamamoto, 1993; Hirai and Nagatsu, 1994; Munchhof and Meyers, 1995; Reding and Buchwald, 1998; Jo et al., 1999; Pachamuthu and Vankar, 2001; Chacko and Ramapanicker, 2015). Using our strategy, we were able to synthesized $N$-Boc-conine $\mathbf{5}$ in optically pure form in few steps from 1-azoniabicyclo[4.1.0] heptane tosylate 2, which was easily access from commercially available chiral aziridine (Choi et al., 2017) (Scheme 2). 


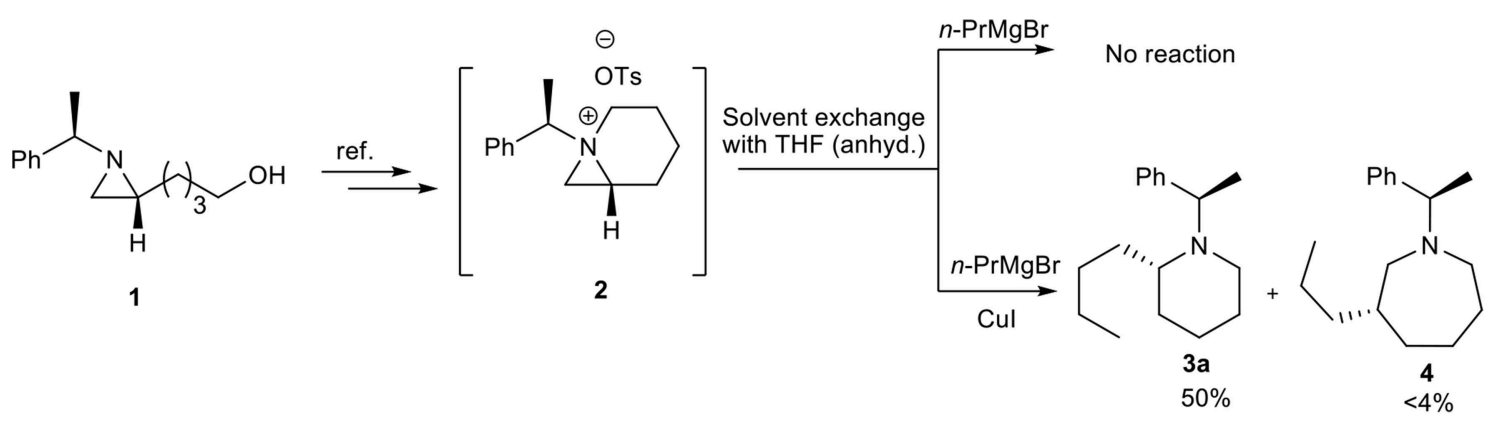

SCHEME 1 | Regioselective synthesis of 2-alkyl substituted piperidine.

TABLE 1 | Optimization of reaction conditions for ring expansion of aziridinium ion with in situ generated organocopper reagents.

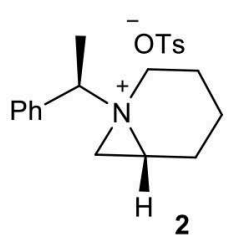

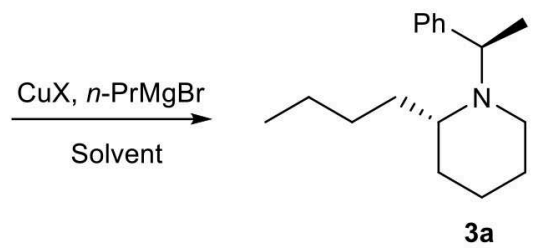

$3 \mathbf{a}$

\begin{tabular}{lllccc}
\hline Entry $^{\mathbf{a}}$ & Reagent $^{\mathbf{b}}$ & Solvent & Temp. ( $\left.{ }^{\circ} \mathbf{C}\right)$ & Time (h) & Yield (\%) $^{\mathbf{c}}$ \\
\hline a & CuCN & THF & $0-25$ & 1.0 & 15 \\
b & CuCN & 1,4-dioxane & $0-25$ & 1.0 & 24 \\
C & CuBr & THF & $0-25$ & 1.0 & 20 \\
d & Cul & 1,4-dioxane & $0-25$ & 1.0 & 70 \\
e & CuBr & 1,4-dioxane & $0-25$ & 1.0 & 32 \\
f & Cul & THF & $0-25$ & 1.0 & 50
\end{tabular}

${ }^{a}$ Reaction was performed at $0.5 \mathrm{mmol} .{ }^{b}$ Copper reagent 1.5 equiv., Grignard 3.0 equiv. ${ }^{c}$ Yield refers to pure products.

Synthesis of $\mathrm{N}$-Boc-conine $\mathbf{5}$ was initiated by the preparation of bicyclic aziridinium ion 2 (Choi et al., 2017) followed by its ring expansion with $\mathrm{EtMgBr}$ and $\mathrm{CuI}$ in 1,4-dioxane as solvent to yield the compound $\mathbf{3 b}$ in $64 \%$ yield in two steps. Onepot debenzylation followed by Boc-protection furnished $N$-Bocconine 5 in $70 \%$ yield whose Boc group would be removed by the known procedure yielding the natural product conine $\mathbf{6}$. Analytical data for compound $\mathbf{5}$ were found in good agreement with reported value in literature (Hirai and Nagatsu, 1994; Jo et al., 1999).

The synthesis of (+)-epiquinamide 7 was the next task by use of our newly developed protocol with the chain extension of $\mathrm{C} 2$ of piperidine. Epiquinamide 7 is a quinolizidine alkaloid isolated from skin of Epipedobates tricolor, an Ecuadorian poisonous frog. It has been found to be highly selective toward $\beta 2$ nicotinic acetylcholine receptors (nAChRs) involving in cellular signaling in both the central (CNS) and peripheral nervous systems (PNS). The importance of this receptor encourages us to utilize our strategy for its synthesis. The formal synthesis of $(+)$-epiquinamide 7 began with our previously prepared hydroxybutyl aziridine $\mathbf{9}$ from aziridine carboxylate $\mathbf{8}$ (Scheme 3) (Choi et al., 2017).
Tosylation of compound 9 with $p$-toluenesulphonic anhydride and triethylamine in dry $\mathrm{CH}_{2} \mathrm{Cl}_{2}$ gives corresponding tosylate $\mathbf{1 0}$ in quantitative yields. Compound 10 was kept in dry $\mathrm{CH}_{3} \mathrm{CN}$ under $\mathrm{N}_{2}$ atmosphere for $24 \mathrm{~h}$ to attain complete conversion to bicyclic aziridinium ion 11, which was confirmed by NMR spectrum. Aziridine ring expansion of 11 with Gilman's reagent generated from Grignard reagent 12 and $\mathrm{CuI}$ gives piperidine $\mathbf{1 3}$ in $57 \%$ yields.

Compound 13 was treated under acidic condition to retrieve an aldehyde from its protected acetal functionality which was subjected to one-pot reductive amination by catalytic hydrogenation under atmospheric $\mathrm{H}_{2}$ to get the bicyclic compound $\mathbf{1 4}$ in 59\% yield which is able to yield epiquinamide (Scheme 4).

\section{CONCLUSION}

In conclusion, we have developed a new and highly efficient method for the selective formation of 2-alkylpiperidine by the alkylative, regio- and stereospecific ring opening of highly strained bicyclic aziridinium ion with organocopper reagent. This method was applied for the synthesis of important alkaloids including conine and (+)-epiquinamide in highly efficient manner.

\section{EXPERIMENTAL SECTION}

\section{Materials and Methods}

Chiral aziridine-2-carboxylates are available as their menthyl ester from Sigma-Aldrich as reagents and from Imagene Co., Ltd. (http://www.imagene.co.kr/) in bulk quantities. Their corresponding ethyl esters were also available either from transesterification of menthyl ester or from Imagene. Reagents are commercially available. All commercially available reagents were used as received unless stated otherwise. Acetonitrile used to make bicyclic aziridinium ion and 1,4-dioxane used in next reactions are purified and used using calcium hydride. All reactions were carried out under an atmosphere of nitrogen in oven-dried glassware with magnetic stirrer. Reactions were monitored by thin layer chromatography (TLC) with $0.25 \mathrm{~mm}$ E. Merck pre-coated silica gel plates (60 F254). Visualization was accomplished with either UV light, or by immersion in 


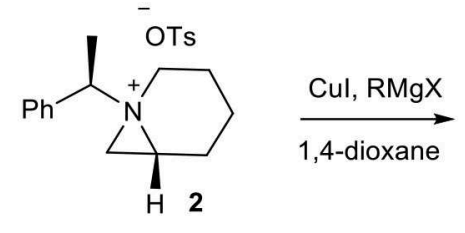<smiles>CCCC1CCCCN1C(C)c1ccccc1</smiles>

3a, $70 \%$<smiles>CCC[C@@H]1CCCCN1[C@@H](C)c1ccccc1</smiles>

3b, $64 \%$<smiles>C[C@H](c1ccccc1)N1CCCC[C@H]1CCc1ccccc1</smiles>

$3 c, 38 \%$<smiles>[R]C[C@@H]1CCCCN1[C@@H](C)c1ccccc1</smiles>

3<smiles>CC[C@@H]1CCCCN1[C@@H](C)c1ccccc1</smiles>

3f, $68 \%$<smiles>C[C@H](c1ccccc1)N1CCCC[C@H]1Cc1ccccc1</smiles>

$3 g, 30 \%$<smiles>Cc1ccccc1</smiles>

3 h, $25 \%$<smiles></smiles>

3d, $33 \%$<smiles>CC(C)C[C@@H]1CCCCN1[C@H](C)c1ccccc1</smiles>

$\mathbf{3 i}, 35 \%$<smiles>C=CCC[C@@H]1CCCCN1[C@@H](C)c1ccccc1</smiles>

$3 e, 50 \%$<smiles>C[C@@H](c1ccccc1)N1CCCC[C@H]1Cc1ccc(Cl)cc1</smiles><smiles>C[C@H](c1ccccc1)N1CCCC[C@H]1CC1CCCC1</smiles>

$3 \mathbf{k}, 27 \%$

solutions of ninhydrin, $p$-anisaldehyde, or phosphomolybdic acid (PMA) followed by heating on a hot plate for about $10 \mathrm{sec}$. Flash column chromatography was carried out using Intertec silica gel (Particle size: 70-230 mesh). ${ }^{1} \mathrm{H}-\mathrm{NMR}$ and ${ }^{13} \mathrm{C}-\mathrm{NMR}$ spectra were obtained using Varian unity INOVA 400WB (400 MHz) or Bruker AVANCE III HD (400 MHz) spectrometer. Chemical shifts are reported relative to chloroform $(\delta=7.26)$ for ${ }^{1} \mathrm{H}$ NMR and chloroform $(\delta=77.2)$ for ${ }^{13} \mathrm{C}$ NMR, acetonitrile $(\delta=1.94)$ for ${ }^{1} \mathrm{H}$ NMR and acetonitrile $(\delta$ $=1.32$ ) for ${ }^{13} \mathrm{C} \mathrm{NMR}$, dimethyl sulfoxide $(\delta=3.33)$ for ${ }^{1} \mathrm{H}$ NMR and dimethyl sulfoxide $(\delta=39.5)$ for ${ }^{13} \mathrm{C}$ NMR. Data are reported as $(\mathrm{br}=$ broad, $\mathrm{s}=$ singlet, $\mathrm{d}=$ doublet, $\mathrm{t}=$ triplet, $\mathrm{q}=$ quartet, $\mathrm{p}=$ quintet, $\mathrm{m}=$ multiplet). Coupling constants are given in $\mathrm{Hz}$. Ambiguous assignments were resolved on the basis of standard one dimensional proton decoupling experiments. Optical rotations were obtained using Rudolph Autopol III digital polarimeter and JASCO P-2000.Optical rotation data was reported as follows: $[\alpha]^{20}$ (concentration $c=$ $\mathrm{g} / 100 \mathrm{~mL}$, solvent). High resolution mass spectra were recorded on a 4.7 Tesla IonSpec ESI-TOFMS, JEOL (JMS-700) and AB Sciex 4800 Plus MALDI TOF ${ }^{\mathrm{TM}}$ (2,5-dihydroxybenzoic acid (DHB) matrix was used to prepare samples for MS. Data was obtained in the reflector positive mode with internal standards for calibration) (Supplementary Materials).

\section{$(6 R)$-1-[(R)-1-Phenylethyl)-1- \\ Azoniabicyclo[4.1.0]Heptane Tosylate (2)}

$p$-Toluenesulfonic anhydride $(489 \mathrm{mg}, 1.50 \mathrm{mmol}$ ) was added to a stirring solution of alcohol $1(300 \mathrm{mg}, 1.36 \mathrm{mmol})$ and $\mathrm{Et}_{3} \mathrm{~N}$ $(0.42 \mathrm{~mL}, 3.0 \mathrm{mmol})$ in $\mathrm{CH}_{2} \mathrm{Cl}_{2}$ at $0^{\circ} \mathrm{C}$. The resulting mixture was stirred for $45 \mathrm{~min}$, warmed to RT, and stirred it for a further additional $30 \mathrm{~min}$. The reaction mixture was quenched with water $(15 \mathrm{~mL})$, extracted with $\mathrm{CH}_{2} \mathrm{Cl}_{2}(3 \times 50 \mathrm{~mL})$, dried over anhydrous $\mathrm{Na}_{2} \mathrm{SO}_{4}$, and concentrated under vacuum to afford the crude tosylate, purified by column chromatography to give pure tosylate as a viscous liquid ( $486 \mathrm{mg}$, 95\% yield).

Freshly prepared tosylate was stored in $\mathrm{CD}_{3} \mathrm{CN}$ for $24 \mathrm{~h}$ to allow complete conversion into azoniabicycle 2 , which was characterized by NMR spectroscopy.

${ }^{1} \mathrm{H}$ NMR $\left(400 \mathrm{MHz}, \mathrm{CD}_{3} \mathrm{CN}\right) \delta 7.61$ (d, $\left.J=8.1 \mathrm{~Hz}, 2 \mathrm{H}\right), 7.63-$ $7.48(\mathrm{~m}, 5 \mathrm{H}), 7.15(\mathrm{~d}, J=7.8 \mathrm{~Hz}, 2 \mathrm{H}), 4.11(\mathrm{q}, J=6.9 \mathrm{~Hz}, 1 \mathrm{H})$, $3.81-3.73(\mathrm{~m}, 1 \mathrm{H}), 3.49(\mathrm{dt}, J=13.3,5.3 \mathrm{~Hz}, 1 \mathrm{H}), 3.23(\mathrm{dd}, J=$ 9.0, $4.2 \mathrm{~Hz}, 1 \mathrm{H}), 3.15$ (ddd, $J=13.4,10.3,4.9 \mathrm{~Hz}, 1 \mathrm{H}), 3.03$ (dd, $J=7.9,4.2 \mathrm{~Hz}, 1 \mathrm{H}), 2.33(\mathrm{~s}, 3 \mathrm{H}), 2.12-2.04(\mathrm{~m}, 2 \mathrm{H}), 1.72(\mathrm{~d}, J=$ 


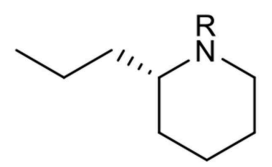

$\mathrm{R}=\mathrm{Boc}, \mathrm{N}-\mathrm{Boc}-$ conine $(\mathbf{5})$ $\mathrm{R}=\mathrm{H},(+)$-Conine $(6)$

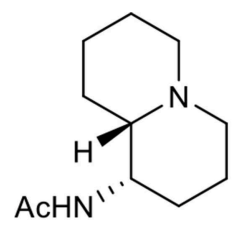

(+)-Epiquinamide (7)
FIGURE $\mathbf{1}$ | Conine $\mathbf{6}$, its Boc-protected form $\mathbf{5}$ and Epiquinamide $\mathbf{7}$

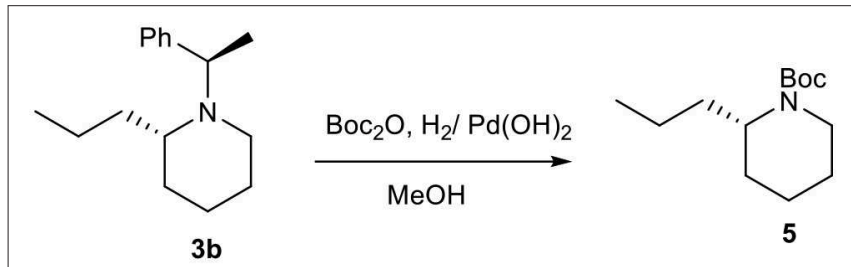

SCHEME 2 | Synthesis of N-Boc-conine $\mathbf{5}$.

$7.0 \mathrm{~Hz}, 3 \mathrm{H}), 1.53-1.44(\mathrm{~m}, 1 \mathrm{H}), 1.37-1.29(\mathrm{~m}, 2 \mathrm{H}), 0.94-0.82(\mathrm{~m}$, $1 \mathrm{H}) ;{ }^{13} \mathrm{C}$ NMR $\left(\mathrm{CD}_{3} \mathrm{CN}, 101 \mathrm{MHz}\right): \delta 146.6,139.6,134.7,131.1$, $130.2,129.5,129.3,126.6,71.7,49.7,49.4,43.1,21.2,20.8,20.7$, $15.5,14.1$.

\section{Typical Procedure for the Synthesis of Various Piperidine (3)}

To a stirred solution of alcohol 1 (200 mg, $0.913 \mathrm{mmol})$ and $\mathrm{Et}_{3} \mathrm{~N}(0.28 \mathrm{~mL}, 0.570 \mathrm{mmol})$ in $\mathrm{CH}_{2} \mathrm{Cl}_{2}(4 \mathrm{~mL})$ at $0^{\circ} \mathrm{C}$ was added $p$-toluenesulfonic anhydride $(328 \mathrm{mg}, 1.00 \mathrm{mmol})$ and the resulting mixture was stirred for another $45 \mathrm{~min}$ and then warmed to $\mathrm{rt}$ and allowed to stir for $30 \mathrm{~min}$. Reaction mixture was concentrated under vacuums and crude tosylate was dissolved in $\mathrm{MeCN}(4.0 \mathrm{~mL})$ and allowed for complete conversion to azoniabicycle 2 for $24 \mathrm{~h}$. Removal of $\mathrm{MeCN}$ solvent from tosylated azonia 2 , added dry 1,4-dioxane $(5 \mathrm{~mL})$ followed by $\mathrm{CuI}(1.5 \mathrm{eq})$ and Grignard reagent (3.0 eq) at $0^{\circ} \mathrm{C}$. The reaction mixture was allowed to stir for appropriate time. After completion of starting material as confirmed by TLC, the reaction mixture was quenched with water $(5 \mathrm{~mL})$. Combined reaction mixture were filtered over Celite, followed by extracted with EtOAc $(2 \times 15 \mathrm{~mL})$, dried over anhydrous $\mathrm{Na}_{2} \mathrm{SO}_{4}$, and concentrated in vacuo to get crude product, which was easily purified by silica gel chromatography to get pure alkylated compound 3 .

\section{(S)-2-Butyl-1-((R)-1-Phenylethyl)Piperidine}

\section{(3a)}

$[\alpha]^{20}{ }_{\mathrm{D}}=+51.2\left(c=0.7, \mathrm{CHCl}_{3}\right) ;{ }^{1} \mathrm{H}$ NMR $\left(400 \mathrm{MHz}, \mathrm{CDCl}_{3}\right)$ $\delta 7.33-7.16(\mathrm{~m}, 5 \mathrm{H}), 4.04(\mathrm{q}, J=6.8 \mathrm{~Hz}, 1 \mathrm{H}), 2.74(\mathrm{ddd}$, $J=11.5,5.6,2.1 \mathrm{~Hz}, 1 \mathrm{H}), 2.41-2.32(\mathrm{~m}, 1 \mathrm{H}), 2.25$ (ddd, $J$ $=16.7,10.2,6.8 \mathrm{~Hz}, 1 \mathrm{H}), 1.66-1.51(\mathrm{~m}, 5 \mathrm{H}), 1.41-1.33(\mathrm{~m}$, $4 \mathrm{H}), 1.31-1.19(\mathrm{~m}, 5 \mathrm{H}), 1.14-1.01(\mathrm{~m}, 1 \mathrm{H}), 0.92-0.84(\mathrm{t}, J=$ $7.0 \mathrm{~Hz}, 3 \mathrm{H}) ;{ }^{13} \mathrm{C}$ NMR $\left(101 \mathrm{MHz}, \mathrm{CDCl}_{3}\right) \delta 143.57,128.03$,
126.64, 57.51, 56.06, 44.56, 29.41, 28.27, 28.01, 25.78, 23.31, 21.88, 21.04, 14.33; HRMS-MALDI $(m / z)$ : calcd. For $\mathrm{C}_{17} \mathrm{H}_{27} \mathrm{~N}$ $[M+\mathrm{H}]^{+}$246.1426; found:246.1422.

\section{(S)-1-((R)-1-Phenylethyl)-2- Propylpiperidine \\ (3b)}

$[\alpha]^{20} \mathrm{D}=+48.8\left(c=0.45, \mathrm{CHCl}_{3}\right) ;{ }^{\mathbf{1}} \mathbf{H}$ NMR $\left(400 \mathrm{MHz}, \mathrm{CDCl}_{3}\right)$ $\delta 7.32-7.22(\mathrm{~m}, 5 \mathrm{H}), 4.03(\mathrm{q}, J=6.8 \mathrm{~Hz}, 1 \mathrm{H}), 2.78-2.67(\mathrm{~m}$, $1 \mathrm{H}), 2.44-2.35(\mathrm{~m}, 1 \mathrm{H}), 2.32-2.22(\mathrm{~m}, 1 \mathrm{H}), 1.66-1.41(\mathrm{~m}, 6 \mathrm{H})$, $1.40-1.33$ (m, 3H), 1.31-1.19 (m, 3H), 1.11 (dddd, $J=20.2,13.1$, 8.3, $5.0 \mathrm{~Hz}, 1 \mathrm{H}), 0.87(\mathrm{t}, J=7.3 \mathrm{~Hz}, 3 \mathrm{H}) ;{ }^{13} \mathrm{C}$ NMR $(101 \mathrm{MHz}$, $\left.\mathrm{CDCl}_{3}\right) \delta 143.71,128.02,126.64,57.54,55.77,44.47,30.57,29.32$, 25.73, 21.80, 21.07, 19.29, 14.68; HRMS-MALDI $(\mathrm{m} / z)$ : calcd. For $\mathrm{C}_{16} \mathrm{H}_{25} \mathrm{~N}[M+\mathrm{H}]^{+}$232.1310; found:232.1319.

\section{(R)-2-Phenethyl-1-((R)-1- \\ Phenylethyl)Piperidine (3c)}

$[\alpha]^{20} \mathrm{D}=+96.3\left(c=0.65, \mathrm{CHCl}_{3}\right) ;{ }^{1} \mathbf{H}$ NMR $\left(400 \mathrm{MHz}, \mathrm{CDCl}_{3}\right)$ $\delta 7.36-7.11(\mathrm{~m}, 10 \mathrm{H}), 4.02(\mathrm{q}, J=6.8 \mathrm{~Hz}, 1 \mathrm{H}), 2.71(\mathrm{~m}, 1 \mathrm{H})$, 2.61-2.50 (m, 2H), 2.44-2.31 (m, 2H), 2.01-1.80 (m, 2H), 1.61 (m, 3H), $1.51-1.38(\mathrm{~m}, 2 \mathrm{H}), 1.36(\mathrm{~d}, J=6.8 \mathrm{~Hz}, 3 \mathrm{H}) ;{ }^{13} \mathrm{C}$ NMR $\left(101 \mathrm{MHz}, \mathrm{CDCl}_{3}\right) \delta 143.82,143.18,128.45,128.42,128.12$, $127.94,126.71,125.74,57.91,55.37,44.29,32.54,30.19,29.01$, 25.46, 21.53, 21.28; HRMS-MALDI $(\mathrm{m} / z)$ : calcd. For $\mathrm{C}_{21} \mathrm{H}_{27} \mathrm{~N}$ $[M+\mathrm{H}]^{+}$294.1345; found:294.1346.

\section{(S)-2-Decyl-1-((R)-1-Phenylethyl)Piperidine (3d)}

$[\alpha]^{20} \mathrm{D}=+68.1\left(c=0.1, \mathrm{CHCl}_{3}\right) ;{ }^{1} \mathbf{H}$ NMR $\left(400 \mathrm{MHz}, \mathrm{CDCl}_{3}\right)$ $\delta$ 7.37-7.26 (m, 5H), $4.06(\mathrm{q}, J=6.8 \mathrm{~Hz}, 1 \mathrm{H}), 2.75(\mathrm{~m}, 1 \mathrm{H})$, $2.39(\mathrm{~s}, 1 \mathrm{H}), 2.34-2.23(\mathrm{~m}, 1 \mathrm{H}), 1.63-1.55(\mathrm{~m}, 5 \mathrm{H}), 1.39(\mathrm{~d}, J=$ $6.7 \mathrm{~Hz}, 3 \mathrm{H}), 1.31(\mathrm{~m}, 18 \mathrm{H}), 1.13(\mathrm{~m}, 1 \mathrm{H}), 0.94-0.86(\mathrm{t}, J=6.8$ $\mathrm{Hz}, 3 \mathrm{H}) ;{ }^{13} \mathrm{C}$ NMR $\left(101 \mathrm{MHz}, \mathrm{CDCl}_{3}\right) \delta 143.57,128.09,126.67$, 56.10, 44.61, 32.08, 30.26, 29.81, 29.52, 28.33, 25.98, 25.81, 22.85, $21.94,21.00,14.26$; HRMS-MALDI $(\mathrm{m} / z)$ : calcd. For $\mathrm{C}_{23} \mathrm{H}_{39} \mathrm{~N}$ $[M+\mathrm{H}]^{+}$330.2336; found:330.2334.

\section{(R)-2-(But-3-En-1-YI)-1-((R)-1- Phenylethyl)Piperidine (3e)}

$[\alpha]^{\mathbf{2 0}} \mathrm{D}=+58.8\left(c=1.2, \mathrm{CHCl}_{3}\right) ;{ }^{1} \mathbf{H} \mathbf{~ N M R}\left(400 \mathrm{MHz}, \mathrm{CDCl}_{3}\right) \delta$ $7.32-7.19(\mathrm{~m}, 5 \mathrm{H}), 5.87-5.70(\mathrm{~m}, 1 \mathrm{H}), 5.04-4.85(\mathrm{~m}, 2 \mathrm{H}), 4.04-$ $3.95(\mathrm{~m}, 1 \mathrm{H}), 2.77-2.64(\mathrm{~m}, 1 \mathrm{H}), 2.51-2.42(\mathrm{~m}, 1 \mathrm{H}), 2.32(\mathrm{ddd}$, $J=15.9,9.4,5.9 \mathrm{~Hz}, 1 \mathrm{H}), 2.07-1.95(\mathrm{~m}, 1 \mathrm{H}), 1.92-1.81(\mathrm{~m}$, $1 \mathrm{H}), 1.80-1.68(\mathrm{~m}, 1 \mathrm{H}), 1.66-1.38(\mathrm{~m}, 5 \mathrm{H}), 1.35(\mathrm{~d}, J=6.8 \mathrm{~Hz}$, $3 \mathrm{H}), 1.34-1.23(\mathrm{~m}, 2 \mathrm{H}) ;{ }^{13} \mathrm{C}$ NMR $\left(101 \mathrm{MHz}, \mathrm{CDCl}_{3}\right) \delta 144.00$, $139.46,128.20,128.07,126.81,114.30,57.95,55.34,44.42,30.53$, 29.02, 27.55, 25.52, 21.68, 21.31; HRMS-MALDI $(\mathrm{m} / z)$ : calcd. For $\mathrm{C}_{17} \mathrm{H}_{25} \mathrm{~N}[M+\mathrm{H}]^{+}$244.1270; found:244.1272.

\section{(S)-2-Ethyl-1-((R)-1-Phenylethyl)Piperidine (3f) $[\alpha]^{20}{ }_{\mathrm{D}}=+63.4\left(c=0.62, \mathrm{CHCl}_{3}\right) ;{ }^{1} \mathbf{H}$ NMR $(400 \mathrm{MHz}$, $\left.\mathrm{CDCl}_{3}\right) \delta 7.34-7.18(\mathrm{~m}, 5 \mathrm{H}), 4.06(\mathrm{q}, J=6.8 \mathrm{~Hz}, 1 \mathrm{H}), 2.79$ $2.71(\mathrm{~m}, 1 \mathrm{H}), 2.32-2.15(\mathrm{~m}, 2 \mathrm{H}), 1.72-1.43(\mathrm{~m}, 6 \mathrm{H}), 1.38(\mathrm{~d}$, $J=6.8 \mathrm{~Hz}, 3 \mathrm{H}), 1.27-1.13(\mathrm{~m}, 2 \mathrm{H}), 0.79(\mathrm{t}, J=7.5 \mathrm{~Hz}, 3 \mathrm{H})$;}


<smiles>C#CCOC(=O)C1CN1[C@@H](C)c1ccccc1</smiles><smiles>C[C@H](c1ccccc1)N1CCC[C@H](O[C@H]2CCCCO2)[C@@H]1CCCC1OCCCO1</smiles>

SCHEME 3 | Synthesis of advance intermediate $\mathbf{1 3}$<smiles>CC(c1ccccc1)N1CC[C@@H](O)[C@H](CCCC2OCCCO2)[C@H]1Cc1ccccc1</smiles>

13

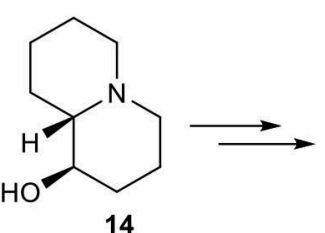

(+)-Epiquinamide (7)

SCHEME 4 | Formal synthesis of (+)-Epiquinamide (7).

${ }^{13} \mathrm{C}$ NMR $\left(101 \mathrm{MHz}, \mathrm{CDCl}_{3}\right) \delta 143.14,128.10,127.99,126.65$, $57.45,57.27,44.73,29.03,25.88,22.14,21.33,20.74,10.23$; HRMS-MALDI $(\mathrm{m} / \mathrm{z})$ : calcd. For $\mathrm{C}_{15} \mathrm{H}_{23} \mathrm{~N}[M+\mathrm{H}]^{+} 218.1153$; found:218.1156.

\section{$(R)-2-B e n z y l-1-((R)-1-$}

\section{Phenylethyl)Piperidine (3g)}

$[\boldsymbol{\alpha}]^{2 \mathbf{2 0}} \mathrm{D}=-8.8\left(c=1.05, \mathrm{CHCl}_{3}\right) ;{ }^{1} \mathbf{H}$ NMR $\left(400 \mathrm{MHz}, \mathrm{CDCl}_{3}\right)$ $\delta 7.36(\mathrm{dt}, J=14.9,4.5 \mathrm{~Hz}, 4 \mathrm{H}), 7.29-7.22(\mathrm{~m}, 1 \mathrm{H}), 7.19-7.06$ $(\mathrm{m}, 3 \mathrm{H}), 6.85(\mathrm{~d}, J=7.0 \mathrm{~Hz}, 2 \mathrm{H}), 4.02(\mathrm{q}, J=6.6 \mathrm{~Hz}, 1 \mathrm{H}), 3.04$ (dd, $J=12.7,3.3 \mathrm{~Hz}, 1 \mathrm{H}), 2.79-2.58(\mathrm{~m}, 4 \mathrm{H}), 1.65-1.60(\mathrm{~m}, 2 \mathrm{H})$, $1.39(\mathrm{~d}, J=6.6 \mathrm{~Hz}, 3 \mathrm{H}), 1.36-1.29(\mathrm{~m}, 2 \mathrm{H}), 1.28-1.22(\mathrm{~m}, 2 \mathrm{H})$; ${ }^{13} \mathrm{C}$ NMR $\left(101 \mathrm{MHz}, \mathrm{CDCl}_{3}\right) \delta 144.79,141.17,129.30,128.44$, $128.31,127.93,126.98,125.70,59.67,57.57,44.25,31.71,27.91$, 26.29, 22.14, 20.51; HRMS-MALDI $(\mathrm{m} / z)$ : calcd. For $\mathrm{C}_{20} \mathrm{H}_{25} \mathrm{~N}$ $[M+\mathrm{H}]^{+}$280.1245; found:280.1240.

\section{(S)-2-Pentyl-1-((R)-1-Phenylethyl)Piperidine}

\section{(3h)}

$[\alpha]^{20} \mathrm{D}=+53.2\left(c=0.65, \mathrm{CHCl}_{3}\right) ;{ }^{1} \mathrm{H}$ NMR $\left(400 \mathrm{MHz}, \mathrm{CDCl}_{3}\right)$ $\delta 7.33-7.19(\mathrm{~m}, 5 \mathrm{H}), 4.04(\mathrm{q}, J=6.8 \mathrm{~Hz}, 1 \mathrm{H}), 2.72(\mathrm{~m}, 1 \mathrm{H})$, 2.39-2.34 (m, $1 \mathrm{H}), 2.32-2.23(\mathrm{~m}, 1 \mathrm{H}), 1.62-1.50(\mathrm{~m}, 4 \mathrm{H}), 1.36$ (d, $J=6.8 \mathrm{~Hz}, 3 \mathrm{H}), 1.33-1.06(\mathrm{~m}, 10 \mathrm{H}), 0.87(\mathrm{t}, J=7.0 \mathrm{~Hz}$, $3 \mathrm{H}) ;{ }^{13} \mathrm{C}$ NMR $\left(101 \mathrm{MHz}, \mathrm{CDCl}_{3}\right) \delta 143.62,128.06,128.03$, $126.66,57.62,56.09,44.58,32.49,29.42,28.25,25.80,25.69,22.82$, $21.89,21.03,14.22$; HRMS-MALDI $(\mathrm{m} / z)$ : calcd. For $\mathrm{C}_{18} \mathrm{H}_{29} \mathrm{~N}$ $[M+\mathrm{H}]^{+}$260.1593; found:260.1594.
(R)-2-Isobutyl-1-((R)-1-

\section{Phenylethyl)Piperidine (3i)}

$[\boldsymbol{\alpha}]^{20} \mathbf{D}=+41.2\left(c=1.1, \mathrm{CHCl}_{3}\right) ;{ }^{1} \mathbf{H}$ NMR $\left(400 \mathrm{MHz}, \mathrm{CDCl}_{3}\right)$ $\delta$ 7.36-7.17 (m, 5H), $3.97(\mathrm{q}, J=6.7 \mathrm{~Hz}, 1 \mathrm{H}), 2.69-2.60(\mathrm{~m}$, $2 \mathrm{H}), 2.46(\mathrm{~m}, 1 \mathrm{H}), 1.70-1.40(\mathrm{~m}, 6 \mathrm{H}), 1.39-1.23(\mathrm{~m}, 6 \mathrm{H}), 0.87$ $(\mathrm{d}, J=6.2 \mathrm{~Hz}, 3 \mathrm{H}), 0.69(\mathrm{~d}, J=6.1 \mathrm{~Hz}) ;{ }^{13} \mathrm{C}$ NMR $(101$ $\left.\mathrm{MHz}, \mathrm{CDCl}_{3}\right) \delta 145.15,128.11,127.82,126.61,58.30,52.98$, $43.73,35.98,28.45,25.28,25.24,24.19,21.99,21.93,20.81$; HRMS-MALDI $(m / z)$ : calcd. For $\mathrm{C}_{17} \mathrm{H}_{27} \mathrm{~N}[M+\mathrm{H}]^{+} 246.1476$; found:246.1479.

\section{(R)-2-Isopentyl-1-((R)-1-}

Phenylethyl)Piperidine (3j)

$[\alpha]^{20}{ }_{\mathrm{D}}=+52.3\left(c=2.35, \mathrm{CHCl}_{3}\right) ;{ }^{1} \mathrm{H}$ NMR $\left(400 \mathrm{MHz}, \mathrm{CDCl}_{3}\right)$ $\delta$ 7.31-7.19 (m, 5H), $4.03(\mathrm{q}, J=6.8 \mathrm{~Hz}, 1 \mathrm{H}), 2.77-2.69(\mathrm{~m}$, $1 \mathrm{H}), 2.38-2.24(\mathrm{~m}, 2 \mathrm{H}), 1.65-1.37(\mathrm{~m}, 7 \mathrm{H}), 1.36(\mathrm{~d}, J=6.8 \mathrm{~Hz}$, $3 \mathrm{H}), 1.30-1.19(\mathrm{~m}, 2 \mathrm{H}), 1.12$ (dddd, $J=16.7,11.9,6.8,5.2 \mathrm{~Hz}$, $1 \mathrm{H}), 0.96(\mathrm{tdd}, J=9.1,5.0,4.2 \mathrm{~Hz}, 1 \mathrm{H}), 0.88(\mathrm{dd}, J=6.7$, $2.7 \mathrm{~Hz}, 6 \mathrm{H}) ;{ }^{13} \mathrm{C}$ NMR $\left(101 \mathrm{MHz}, \mathrm{CDCl}_{3}\right) \delta 143.60,128.02$, $126.64,57.68,56.28,44.60,35.21,29.40,28.61,25.87,25.81,22.94$, $22.74,21.86,21.01$; HRMS-MALDI $(\mathrm{m} / \mathrm{z})$ : calcd. For $\mathrm{C}_{18} \mathrm{H}_{29} \mathrm{~N}$ $[M+\mathrm{H}]^{+}$260.1523; found:260.1526.

\section{(R)-2-(4-Chlorobenzyl)-1-((R)-1- Phenylethyl)Piperidine (3k)}

$[\boldsymbol{\alpha}]^{20}{ }_{\mathrm{D}}=+3.6\left(c=1.28, \mathrm{CHCl}_{3}\right) ;{ }^{1} \mathbf{H}$ NMR $(400 \mathrm{MHz}$, $\left.\mathrm{CDCl}_{3}\right) \delta 7.40-7.23(\mathrm{~m}, 5 \mathrm{H}), 7.16-7.08(\mathrm{~m}, 2 \mathrm{H}), 6.75(\mathrm{~d}$, 
$J=8.3 \mathrm{~Hz}, 2 \mathrm{H}), 3.97(\mathrm{q}, J=6.6 \mathrm{~Hz}, 1 \mathrm{H}), 2.95(\mathrm{~m}, 1 \mathrm{H})$, $2.77-2.56(\mathrm{~m}, 4 \mathrm{H}), 1.68-1.53(\mathrm{~m}, 3 \mathrm{H}), 1.38(\mathrm{~d}, J=6.6 \mathrm{~Hz}$, $3 \mathrm{H}), 1.28-1.13(\mathrm{~m}, 3 \mathrm{H}) ;{ }^{13} \mathrm{C}$ NMR $\left(101 \mathrm{MHz}, \mathrm{CDCl}_{3}\right) \delta$ $144.66,139.56,131.36,130.49,128.43,128.33,127.80,127.00$, $59.77,57.34,44.13,30.85,27.63,26.06,22.13,20.28$; HRMSMALDI $(m / z)$ : calcd. For $\mathrm{C}_{20} \mathrm{H}_{24} \mathrm{ClN}[M+\mathrm{H}]^{+}$313.9880; found:313.9882.

\section{(R)-2-(Cyclopentylmethyl)-1-((R)-1- Phenylethyl)Piperidine (3I)}

$[\alpha]^{\mathbf{2 0}} \mathrm{D}=+96.7\left(c=0.395, \mathrm{CHCl}_{3}\right) ;{ }^{\mathbf{1}} \mathbf{H} \mathbf{N M R}(400 \mathrm{MHz}$, $\left.\mathrm{CDCl}_{3}\right) \delta 7.33-7.19(\mathrm{~m}, 5 \mathrm{H}), 3.98(\mathrm{q}, J=6.7 \mathrm{~Hz}, 1 \mathrm{H}), 2.73-$ $2.55(\mathrm{~m}, 2 \mathrm{H}), 2.51-2.41(\mathrm{~m}, 1 \mathrm{H}), 1.82-1.38(\mathrm{~m}, 16 \mathrm{H}), 1.33$ $(\mathrm{d}, J=6.7 \mathrm{~Hz}, 3 \mathrm{H}) 1.15-1.02(\mathrm{~m}, 1 \mathrm{H}) ;{ }^{13} \mathrm{C}$ NMR $(101 \mathrm{MHz}$, $\left.\mathrm{CDCl}_{3}\right) \delta 144.98,128.10,127.83,126.63,58.37,54.33,43.90$, 37.37, 33.99, 33.09, 32.05, 28.57, 25.39, 25.20, 25.00, 21.85, 20.96; HRMS-MALDI $(m / z)$ : calcd. For $\mathrm{C}_{19} \mathrm{H}_{29} \mathrm{~N}[M+\mathrm{H}]^{+}$272.0563; found:272.0562.

\section{(S)-Tert-Butyl \\ 2-Propylpiperidine-1-Carboxylate: (+)-Boc-Coniine (5)}

To a solution of compound $3 \mathbf{b}(0.3 \mathrm{~g}, 1.296 \mathrm{mmol})$ in $\mathrm{MeOH}$. $\mathrm{Pd}(\mathrm{OH})_{2}(0.455 \mathrm{~g}, 2.24 \mathrm{mmol})$ and $\mathrm{Boc}_{2} \mathrm{O}(0.566 \mathrm{~g}, 2.59 \mathrm{mmol})$ were added. The reaction mixture was allowed to stir under hydrogenation atmospheric pressure of hydrogen for $12 \mathrm{~h}$. The reaction mixture was filtered over Celite pad by using $\mathrm{MeOH}$ as solvent, concentrated in vacuo to get crude product which was purified by column chromatography to give pure product 5 (206 mg, $70 \%$ ).

$[\alpha]^{20}{ }_{\mathrm{D}}=+16.072\left(c=1.0, \mathrm{CHCl}_{3}\right) ;{ }^{\mathbf{1}} \mathrm{H}$ NMR $(400 \mathrm{MHz}$, $\left.\mathrm{CDCl}_{3}\right) \delta 4.21(\mathrm{~s}, 1 \mathrm{H}), 3.96(\mathrm{~d}, J=11.9 \mathrm{~Hz}, 1 \mathrm{H}), 2.74(\mathrm{~m}$, $1 \mathrm{H}), 1.65-1.54(\mathrm{~m}, 5 \mathrm{H}), 1.45(\mathrm{~s}, 9 \mathrm{H}), 1.43-1.20(\mathrm{~m}, 5 \mathrm{H}), 0.92$ $(\mathrm{t}, J=7.3 \mathrm{~Hz}, 3 \mathrm{H}) ;{ }^{13} \mathrm{C}$ NMR $\left(101 \mathrm{MHz}, \mathrm{CDCl}_{3}\right) \delta 155.36$, $79.09,50.32,38.83,32.10,28.67,27.59,25.88,19.66,19.23$, 14.24; HRMS-MALDI $(\mathrm{m} / z)$ : calcd. For $\mathrm{C}_{13} \mathrm{H}_{25} \mathrm{NO}_{2}[M+\mathrm{Na}]^{+}$ 250.0227; found:250.0229.

\section{(R)-4-(Benzyloxy)-4-((S)-1-((R)-1- Phenylethyl)Aziridin-2-yl)Butyl-4- Methylbenzenesulfonate (10)}

p-Toluenesulfonic anhydride (300 $\mathrm{mg}, 0.92 \mathrm{mmol})$ was added to a stirring solution of $9(330 \mathrm{mg}, 1.01 \mathrm{mmol})$ and triethylamine $(0.28 \mathrm{~mL}, 2.02 \mathrm{mmol})$ in $\mathrm{CH}_{2} \mathrm{Cl}_{2}$ at $0^{\circ} \mathrm{C}$. The resulting mixture was stirred for $45 \mathrm{~min}$, warmed to $\mathrm{rt}$, and stirred for a further $30 \mathrm{~min}$. The reaction mixture was quenched with water, extracted with $\mathrm{CH}_{2} \mathrm{Cl}_{2}$, dried over sodium sulfate, and concentrated under vacuum to afford the crude tosylate, which was purified by chromatography to give pure tosylate as a viscous liquid 10 (490 mg, 95\% yield).

${ }^{1} \mathrm{H}$ NMR $\left(400 \mathrm{MHz}, \mathrm{CD}_{3} \mathrm{CN}\right) \delta 7.79-7.64(\mathrm{~m}, 2 \mathrm{H}), 7.42-$ $7.39(\mathrm{~m}, 2 \mathrm{H}), 7.36-7.17(\mathrm{~m}, 10 \mathrm{H}), 4.53(\mathrm{~m}, 1 \mathrm{H}), 4.31(\mathrm{~d}$, $J=11.7 \mathrm{~Hz}, 1 \mathrm{H}), 3.71-3.61(\mathrm{~m}, 2 \mathrm{H}), 2.75(\mathrm{td}, J=7.2$, $3.5 \mathrm{~Hz}, 1 \mathrm{H}), 2.43(\mathrm{~s}, 3 \mathrm{H}), 2.42-2.37(\mathrm{~m}, 1 \mathrm{H}), 1.77(\mathrm{~d}, J=$ $3.3 \mathrm{~Hz}, 1 \mathrm{H}), 1.51(\mathrm{~d}, J=6.2 \mathrm{~Hz}, 1 \mathrm{H}), 1.45-1.36(\mathrm{~m}, 2 \mathrm{H})$, $1.34(\mathrm{~d}, J=6.5 \mathrm{~Hz}, 3 \mathrm{H}), 1.31-1.16(\mathrm{~m}, 2 \mathrm{H}), 1.03-0.87(\mathrm{~m}$,
$1 \mathrm{H}) ;{ }^{13} \mathrm{C}$ NMR (101 MHz, CD 3 CN) $\delta 145.86,145.52,139.90$, $133.73,130.72,129.79,129.43,128.92,128.41,128.30,128.04$, $127.88,80.00,71.72,71.37,70.39,40.84,33.76,29.32,24.48$, $22.66,21.42$.

\section{(5R, 6S)-5-(Benzyloxy)-1-((R)-1- Phenylethyl)-1-Azoniabicyclo[4.1.0] Heptane Tosylate (11)}

Freshly prepared tosylate 10 was stored in $\mathrm{CD}_{3} \mathrm{CN}$ for $24 \mathrm{~h}$ to allow complete conversion into azoniabicycle 11, which was characterized by NMR spectroscopy.

${ }^{1} \mathrm{H}$ NMR (400 MHz, CD $\left.3 \mathrm{CN}\right) \delta 7.68-7.63(\mathrm{~m}, 2 \mathrm{H}), 7.61-7.57$ (m, 2H), 7.47-7.27 (m, 9H), $7.15(\mathrm{dd}, J=8.4,0.6 \mathrm{~Hz}, 2 \mathrm{H}), 4.61(\mathrm{~d}$, $J=11.6 \mathrm{~Hz}, 1 \mathrm{H}), 4.53(\mathrm{~d}, J=11.6 \mathrm{~Hz}, 1 \mathrm{H}), 4.31(\mathrm{q}, J=7.0 \mathrm{~Hz}$, $1 \mathrm{H}), 4.15-4.04(\mathrm{~m}, 2 \mathrm{H}), 3.50-3.39(\mathrm{~m}, 2 \mathrm{H}), 3.28-3.14(\mathrm{~m}, 2 \mathrm{H})$, $2.30(\mathrm{~s}, 3 \mathrm{H}), 1.69$ (d, $J=7.0 \mathrm{~Hz}, 3 \mathrm{H}), 1.65-1.47$ (m, 2H), 1.34$1.13(\mathrm{~m}, 2 \mathrm{H}) ;{ }^{13} \mathrm{C}$ NMR $\left(101 \mathrm{MHz}, \mathrm{CD}_{3} \mathrm{CN}\right) \delta 146.34,139.76$, $138.97,134.48,130.93,129.90,129.68,129.37,129.27,128.58$, $128.50,126.61,71.51,69.58,53.04,48.70,42.50,21.85,21.25$, $15.72,14.18$.

\section{(2S,3R)-2-(3-(1,3-Dioxan-2-YI)Propyl)-3- (Benzyloxy)-1-((R)-1- Phenylethyl)Piperidine (13)}

After removal of $\mathrm{MeCN}$ solvent from tosylated azonia 11, dry 1,4-dioxane $(10 \mathrm{~mL})$ followed by $\mathrm{CuI}(1.5 \mathrm{eq})$ and Grignard reagent 12 (3.0 eq) were added and allowed to stir at $0^{\circ} \mathrm{C}$ for $15 \mathrm{~min}$. followed by additional $40 \mathrm{~min}$. at room temperature. After completion of starting material as confirmed by TLC, the reaction mixture was quenched with water $(5 \mathrm{~mL})$. The Combined reaction mixture were filtered over Celite, followed by extracted with EtOAc $(2 \times$ $10 \mathrm{~mL}$ ), dried over anhydrous $\mathrm{Na}_{2} \mathrm{SO}_{4}$, and concentrated in vacuo to get crude product, which was easily purified by silica gel chromatography to get pure alkylated compound 13 (104 mg, $57 \%$ ).

$[\alpha]^{20}{ }_{\mathrm{D}}=+24.012\left(c=0.20, \mathrm{CHCl}_{3}\right) ; \mathbf{1 H}$ NMR $(400 \mathrm{MHz}$, CDCl3) $\delta 7.38-7.26(\mathrm{~m}, 10 \mathrm{H}), 4.66(\mathrm{~d}, J=10.6 \mathrm{~Hz}, 1 \mathrm{H}), 4.60-$ $4.45(\mathrm{~m}, 2 \mathrm{H}), 4.46-4.35(\mathrm{~m}, 1 \mathrm{H}), 4.17-4.07(\mathrm{~m}, 1 \mathrm{H}), 3.97-$ $3.73(\mathrm{~m}, 3 \mathrm{H}), 3.54-3.40(\mathrm{~m}, 1 \mathrm{H}), 2.97-2.83(\mathrm{~m}, 2 \mathrm{H}), 2.81-$ $2.63(\mathrm{~m}, 1 \mathrm{H}), 2.29-2.17(\mathrm{~m}, 1 \mathrm{H}), 2.16-1.77(\mathrm{~m}, 4 \mathrm{H}), 1.66-$ $1.51(\mathrm{~m}, 2 \mathrm{H}), 1.49(\mathrm{~d}, \mathrm{~J}=6.8 \mathrm{~Hz}, 3 \mathrm{H}), 1.44-1.22(\mathrm{~m}, 4 \mathrm{H})$; 13C NMR (101 MHz, CDCl3) $\delta 142.08,138.70,128.42,128.26$, $128.21,127.93,127.56,127.06,127.51,127.48,127.10,102.37$, $74.93,71.02,66.90,60.12,58.02,57.13,43.56,27.52,25.88$, $20.49,19.39$.

\section{(1R, 9aS)-Octahydro-1H-Quinolizin-1-OI (14)}

To a stirred solution of $\mathbf{1 3}$ (100 mg, $0.236 \mathrm{mmol})$ in water $(2 \mathrm{ml})$ treated with catalytic amount of sulfuric acid and stir it for $2 \mathrm{~h}$, followed by quenched with $\mathrm{NaHCO}_{3}$. The aqueous phase was extracted with $\mathrm{CH}_{2} \mathrm{Cl}_{2}$. The combined organic layers were dried over anhydrous $\mathrm{Na}_{2} \mathrm{SO}_{4}$, concentrated in vacuo to get crude product, which was dissolved in $\mathrm{MeOH}(2 \mathrm{ml})$ followed by $\mathrm{Pd}(\mathrm{OH})_{2}(98 \mathrm{mg}, 0.709 \mathrm{mmol})$ was added and the mixture 
was hydrogenated under an atmospheric pressure of hydrogen for $12 \mathrm{~h}$. The reaction mixture was diluted with $\mathrm{MeOH}$ and filtered on a pad of Celite using $\mathrm{MeOH}$ as solvent. The pure product was obtained by column chromatography as viscous liquid 14 (57 mg, 59\%).

$[\alpha]^{20}{ }_{\mathrm{D}}=-20.307 \quad\left(c=0.35, \mathrm{CHCl}_{3}\right) ;{ }^{\mathbf{1}} \mathbf{H}$ NMR $(400$ $\left.\mathrm{MHz}, \mathrm{CDCl}_{3}\right) \delta 4.53$ (s, 1H), 3.39 (ddd, $J=11.1,9.0$, $4.5 \mathrm{~Hz}, 1 \mathrm{H}), 2.96(\mathrm{~d}, J=11.2 \mathrm{~Hz}, 1 \mathrm{H}), 2.84(\mathrm{~d}, J=$ $11.4 \mathrm{~Hz}, 1 \mathrm{H}), 2.25-1.98(\mathrm{~m}, 4 \mathrm{H}), 1.88-1.59$ (m, 6H), 1.37$1.22(\mathrm{~m}, 3 \mathrm{H}) ;{ }^{13} \mathrm{C}$ NMR (101 $\left.\mathrm{MHz}, \mathrm{CDCl}_{3}\right) \delta$ 72.76, $68.92,56.25,56.01,34.12,28.82,25.79,24.28,23.45$; HRMSMALDI $(m / z)$ : calcd. For $\mathrm{C}_{9} \mathrm{H}_{17} \mathrm{NO}[M+\mathrm{H}]^{+}$156.0543; found:156.0546.

\section{DATA AVAILABILITY}

All datasets generated for this study are included in the manuscript and/or the Supplementary Files.

\section{REFERENCES}

Airiau, E., Spangenberg, T., Girard, N., Breit, B., and Mann, A. (2010). Short access to $(+)$-lupinine and (+)-epiquinamide via double hydroformylation. Org. Lett. 12, 528-531. doi: 10.1021/ol902718q

Bailey, P. D., Millwood, P. A., and Smith, P. D. (1998). Asymmetric routes to substituted piperidines. Chem. Commun. 633-640. doi: 10.1039/a709071d

Boydas, E. B., Tanriver, G., D'hooghe, M., Ha, H.-J., Van Speybroeck, V., and Catak, S. (2018). Theoretical insight into the regioselective ring-expansions of bicyclic aziridinium ions. Org. Biomol. Chem. 16, 796-806. doi: 10.1039/C7OB $02253 \mathrm{~K}$

Carry, J. C., Brohan, E., Perron, S., and Bardouillet, P. E. (2013). Chiral supercritical fluid chromatography in the preparation of enantiomerically pure (S)-(+)-tertButyl-3-hydroxyazepane-1-carboxylate. Org. Process Res.Dev.17, 1568-1571. doi: $10.1021 /$ op $400274 \mathrm{~b}$

Chacko, S., and Ramapanicker, R. (2015). Proline catalyzed, one-pot three component Mannich reaction and sequential cyclization toward the synthesis of 2-substituted piperidine and pyrrolidine alkaloids. Tetrahedron Lett. 56, 2023-2026. doi: 10.1016/j.tetlet.2015.03.001

Chavan, S. P., Khairnar, L. B., Pawar, K. P., Chavan, P. N., and Kawale, S. A. (2015). Enantioselective syntheses of (R)-pipecolic acid, (2R, 3R)-3-hydroxypipecolic acid, $\beta$-(+)-conhydrine and (-)-swainsonine using an aziridine derived common chiral synthon. RSC Adv. 5, 50580-50590. doi: 10.1039/C5RA 06429E

Choi, J., Yadav, N. N., and Ha, H. J. (2017). Preparation of a stable bicyclic aziridinium ion and its ring expansion toward piperidines and azepanes. Asian J. Org. Chem. 6, 1292-1307. doi: 10.1002/ajoc.201700080

D'hooghe, M., and Ha, H.-J. (eds). (2016). Synthesis of 4- to 7-Membered Heterocycles by Ring Expansion: Aza-, Oxa-and Thiaheterocyclic Small-Ring Systems. Heidelberg: Springer.

Dolfen, J., Yadav, N. N., De Kimpe, N., D'hooghe, M., and Ha, H. J. (2016). Bicyclic aziridinium ions in azaheterocyclic chemistry - preparation and synthetic application of 1-Azoniabicyclo [n.1.0]alkanes. Adv. Synth. Catal. 358, 3483-3511. doi: 10.1002/adsc.201601081

Eum, H., Choi, J., Cho, C.-G., and Ha, H.-J. (2015). RegiochemistryDirected syntheses of polyhydroxylated alkaloids from chiral aziridines. Asian J. Org. Chem. 4, 1325-1340. doi: 10.1002/ajoc.20150 0380

Ghorai, M. K., Nanaji, Y., and Yadav, A. K. (2011). Ring opening/C-N cyclization of activated aziridines with carbon nucleophiles: highly diastereo- and enantioselective synthesis of tetrahydroquinolines. Org. Lett. 13, 4256-4259. doi: $10.1021 / \mathrm{ol} 2016077$

\section{AUTHOR CONTRIBUTIONS}

All authors listed have made a substantial, direct and intellectual contribution to the work, and approved it for publication.

\section{FUNDING}

This work was supported by the National Research Foundation of Korea (NRF-2012M3A7B4049645 and 2014R1A5A1011165 with Centre for New Directions in Organic Synthesis).

\section{SUPPLEMENTARY MATERIAL}

The Supplementary Material for this article can be found online at: https://www.frontiersin.org/articles/10.3389/fchem. 2019.00460/full\#supplementary-material

Hattori, K., and Yamamoto, H. (1993). Asymmetric aza-Diels-Alder reaction: enantio- and diastereoselective reaction of imine mediated by Chiral Lewis acid. Tetrahedron 49, 1749-1760. doi: 10.1016/S0040-4020(01)80532-9

Hirai, Y., and Nagatsu, M. (1994). Construction of chiral 2-functionalized piperidine via enzymatic resolution and palladium-catalyzed $\mathrm{N}$-alkylation. Chem. Lett. 23, 21-22. doi: 10.1246/cl.1994.21

Husson, H.-P., and Royer, J. (1999). Chiral non-racemic Ncyanomethyloxazolidines: the pivotal system of the $\mathrm{CN}(\mathrm{R}, \mathrm{S})$ method. Chem. Soc. Rev. 28, 383-394. doi: 10.1039/a900153k

Ji, M.-K., Hertsen, D., Yoon, D.-H., Eum, H., Goossens, H., Waroquier, M., et al. (2014). Nucleophile-dependent regio- and stereoselective ring opening of 1-Azoniabicyclo-[3.1.0] hexane tosylate. Chem. Asian. J. 9, 1060-1067. doi: 10.1002/asia.201301551

Jo, E., Na, Y., and Chang, S. (1999). A highly efficient synthesis of (S)-(+)-NBoc-coniine using ring-closing olefin metathesis (RCM). Tetrahedron Lett. 40, 5581-5582. doi: 10.1016/S0040-4039(99)01081-3

Kim, Y., Ha, H.-J., Yun, S. Y., and Lee, W. K. (2008). The preparation of stable aziridinium ions and their ring-openings. Chem. Commun. 4363-4365. doi: 10.1039/b809124b

Kim, Y., Ha, H. J., Yun, H., Lee, B. K., and Lee, W. K. (2006). Ring opening of 2-acylaziridines by acid chlorides. Tetrahedron 62, 8844-8849. doi: $10.1016 /$ j.tet.2006.06.025

Kozlowski, J. A. (1991). Organocuprates in the conjugate addition reaction. Compr. Org. Synth. 4, 169-198. doi: 10.1016/B978-0-08-052349-1.00093-7

Kumar, P., and Bodas, M. S. (2005). Asymmetric synthesis of both the enantiomers of trans-3-Hydroxypipecolic acid. J. Org. Chem. 70, 360-363. doi: $10.1021 /$ jo0 485381

Macha, L., D'hooghe, M., and Ha, H.-J. (2019). Deployment of aziridines for the synthesis of alkaloids and their derivatives. Synthesis 51, 1491-1515. doi: 10.1055/s-0037-1611715

Masse, C. E., Morgan, A. J., and Panek, J. S. (2000). An asymmetric aminohydroxylation approach to the azepine core of (-)-balanol. Org. Lett. 2, 2571-2573. doi: 10.1021/ol0061034

Munchhof, M. J., and Meyers, A. I. (1995). An asymmetric route to chiral, nonracemic 2-substituted piperidines. Synthesis of (-)-Pipecoline, (+)-Coniine, and (-)-Coniceine. J. Org. Chem. 60, 7084-7085. doi: 10.1021/jo00127a005

Nicolaou,K. C., Koide, K., and Bunnage, M. E. (1995). Total synthesis of balanol and designed analogues. Chem. Eur. J. 1, 454-466. doi: 10.1002/chem.19950010711

Pachamuthu, K., and Vankar, Y. D. (2001). Synthesis of (-)-coniine and (-)pipecoline using ruthenium catalyzed ring closing metathesis. J. Organomet. Chem. 624, 359-363. doi: 10.1016/S0022-328X(00)00930-X 
Passarella, D., Angoli, M., Giardini, A., Lesma, G., Silvani, A., and Danieli, B. (2002). Concise total synthesis of $( \pm)$-Aloperine and 6-epi-Aloperine. Org. Lett. 4, 2925-2928. doi: 10.1021/ol0263144

Reding, M. T., and Buchwald, S. L. (1998). Short enantioselective total syntheses of the piperidine Alkaloids (S)- Coniine and (2R,6R)-trans-solenopsin A via catalytic asymmetric imine hydrosilylation. J. Org. Chem. 63, 6344-6347. doi: $10.1021 /$ jo980808q

Srivastava, A. K., Das, S. K., and Panda, G. (2009). An approach towards the total synthesis of $(+)$-epiquinamide and $(+)-\alpha$-conhydrine from Garner aldehyde. Tetrahedron 65, 5322-5327. doi: 10.1016/j.tet.2009. 04.074

Srivastava, A. K., and Panda, G. (2008). Total synthesis of (-)-balanol, all stereoisomers, their $\mathrm{N}$ - tosyl analogues, and fully protected ophiocordin: an easy route to hexahydroazepine cores from garner aldehydes. Chem. Eur. J. 14, 4675-4688. doi: 10.1002/chem.200701991

Stanković, S., D’hooghe, M., Catak, S., Eum, H., Waroquier, M., Van Speybroeck, V., et al. (2012). Regioselectivity in the ring opening of non-activated aziridine. Chem. Soc. Rev. 41, 643-665. doi: 10. 1039/C1CS15140A

Suyama, T. L., and Gerwick, W. H. (2006). Practical total syntheses of epiquinamide enantiomers. Org. Lett. 08, 4541-4543. doi: 10.1021/ol061736p

Tong, S. T., and Barker, D. (2006). A concise synthesis of $( \pm$ ) and a total synthesis of (+)-epiquinamide. Tetrahedron Lett. 47, 5017-5020. doi: 10.1016/j.tetlet.2006.05.092

Trost, B. M., Fandrick, D. R., Brodmann, T., and Stiles, D. T. (2007). Dynamic kinetic asymmetric allylic amination and acyl migration of vinyl aziridines with imido carboxylates. Angew. Chem. Int. Ed. 46, 6123-6125. doi: 10.1002/anie.200700835

Yadav, N. N., Choi, J., and Ha, H. J. (2016). Asymmetric synthesis of 2, 6-cisdisubstituted piperidine alkaloids from chiral aziridine. Org. Biomol. Chem. 14, 6426-6434. doi: 10.1039/C6OB00806B

Yadav, N. N., and Ha, H.-J. (2018). Preparation of stable bicyclic aziridinium ions and their ring-opening for the synthesis of azaheterocycles. J. Vis. Exp. e57572. doi: $10.3791 / 57572$

Yoon, D.-H., Kang, P., Lee, W. K., Kim, Y., and Ha, H.-J. (2012). Alkylative ring opening of $\mathrm{N}$-methyl aziridinium ions and a formal synthesis of tyroscherin. Org. Lett. 14, 429-431. doi: 10.1021/ol202 $683 \mathrm{k}$

Yudin, A. K. (2006). Aziridines and Epoxides in Organic Synthesis. Weinheim: Wiley-VCH.

Conflict of Interest Statement: The authors declare that the research was conducted in the absence of any commercial or financial relationships that could be construed as a potential conflict of interest.

Copyright (c) 2019 Yadav, Lee, Srivastava and Ha. This is an open-access article distributed under the terms of the Creative Commons Attribution License (CC BY). The use, distribution or reproduction in other forums is permitted, provided the original author(s) and the copyright owner(s) are credited and that the original publication in this journal is cited, in accordance with accepted academic practice. No use, distribution or reproduction is permitted which does not comply with these terms. 